\title{
Laser-nucleated acoustic cavitation in focused ultrasound
}

\author{
Bjoern Gerold, ${ }^{1, a)}$ Spiros Kotopoulis, ${ }^{2, a)}$ Craig McDougall, ${ }^{3}$ David McGloin, ${ }^{3}$ \\ Michiel Postema, ${ }^{2,4}$ and Paul Prentice ${ }^{1}$ \\ ${ }^{1}$ Institute for Medical Science and Technology, The University of Dundee, Dundee DD2 1FD, United Kingdom \\ ${ }^{2}$ Department of Engineering, The University of Hull, Kingston upon Hull HU6 7RX, United Kingdom \\ ${ }^{3}$ Division of Electronic Engineering and Physics, The University of Dundee, \\ Dundee DD1 4HN, United Kingdom \\ ${ }^{4}$ Department of Physics and Technology, University of Bergen, Allégaten 55, 5007 Bergen, Norway
}

(Received 6 February 2011; accepted 22 March 2011; published online 26 April 2011)

\begin{abstract}
Acoustic cavitation can occur in therapeutic applications of high-amplitude focused ultrasound. Studying acoustic cavitation has been challenging, because the onset of nucleation is unpredictable. We hypothesized that acoustic cavitation can be forced to occur at a specific location using a laser to nucleate a microcavity in a pre-established ultrasound field. In this paper we describe a scientific instrument that is dedicated to this outcome, combining a focused ultrasound transducer with a pulsed laser. We present high-speed photographic observations of laser-induced cavitation and lasernucleated acoustic cavitation, at frame rates of $0.5 \times 10^{6}$ frames per second, from laser pulses of energy above and below the optical breakdown threshold, respectively. Acoustic recordings demonstrated inertial cavitation can be controllably introduced to the ultrasound focus. This technique will contribute to the understanding of cavitation evolution in focused ultrasound including for potential therapeutic applications. (C) 2011 American Institute of Physics. [doi:10.1063/1.3579499]
\end{abstract}

\section{INTRODUCTION}

Cavitation is the formation of cavities or bubbles in liquids. Apart from mechanical or hydraulic cavitation, which occur in a rapidly flowing fluid, cavities can be generated using high-amplitude acoustics or optically induced via absorption of a laser pulse focused into a liquid. Acoustic and optical cavitations both occur above some characteristic threshold intensity for the respective forms of radiation. For the former, the threshold is a function of the peak-negative pressure (PNP) and frequency of the ultrasound, and is sometimes referred to as the Blake threshold. ${ }^{1}$ The mechanical index (MI) is a qualitative indication of the likelihood of cavitation occurring during a given ultrasound exposure, and is given by: ${ }^{2}$

$$
\mathrm{MI}=\frac{\mathrm{PNP}}{\sqrt{f}},
$$

where PNP is the peak-negative pressure normalized by $1 \mathrm{MPa}$ and $f$ is the center frequency of the ultrasound field normalized by $1 \mathrm{MHz}$. Acoustic cavitation is perhaps best known for its role in ultrasonic cleaning, but it is also encountered during the application of focused ultrasound surgery (FUS). FUS procedures involve high-intensity focused ultrasound (HIFU) in the $\mathrm{MHz}$ range onto target tissue such as a tumor, for ablation via viscous absorption of the mechanical energy. This has the distinct advantage of avoiding any requirement for invasive surgical intervention. Cavitation is currently carefully avoided in clinical FUS procedures, as it acts to scatter incident ultrasound radiation, which may result in malformed and unpredictable lesions. However, it is under investigation for potential enhancement of therapeutic

a) These authors contributed equally to this work. effects during FUS including as a mechanism for rapid heat deposition, and for actively promoting drug delivery via tissue disruption. ${ }^{3,4}$ Distinguishing between the types of acoustic cavitation, ${ }^{5}$ is useful for considering the related effects that may be harnessed for therapeutic applications. Stable (non inertial) cavitation refers to extended periodic oscillations around some equilibrium radius, which generate acoustic emissions at the driving frequency and its harmonics. Transient (or inertial) cavitation describes unstable growth followed by rapid collapse, driven by the inertia of the host medium. This form of cavitation is associated with high localized energy densities and broadband acoustic emissions, ${ }^{6}$ and may be monitored by way of a dose-control mechanism for possible enhanced FUS effects.

Current understanding of cavitation in focused ultrasound is hampered by inherent difficulties in studying the phenomenon, including ultrafast dynamics at typical HIFU frequencies and the small cavitation nuclei, which are a few micrometers. Moreover, the exact site of nucleation is difficult to predict, often occurring at an impurity or gaseous inclusion within the field. Addressing some of these issues provided the motivation for the work reported here. In contrast, laser-induced cavitation, whereby a short laser pulse is focused into a liquid to form a plasma which rapidly expands to form a cavity, ${ }^{7,8}$ is a well-established and understood approach used to study single-cavity dynamics in a range of fluidic environments. ${ }^{9}$ The distinct advantage of this technique is the predetermined location of the cavity, defined by the point to which the laser pulse is focused. This allows the incorporation of high-speed cameras, capable of imaging the dynamics at frame rates capable of resolving these dynamics. In this manner, cavitation-related activity such as the phenomenon of jet formation from collapsing cavities in the vicinity of a boundary, ${ }^{10}$ causing material erosion and 
surface cleaning, can be reliably and reproducibly investigated. The uncertainty of the positional and temporal occurrence of acoustic cavitation prevents meaningful implementation of high-speed cameras for studying the development of cavitation activity in ultrasound fields. A number of such studies have been attempted, ${ }^{11,12}$ although the large field-ofview and long exposure times, the investigators were required to employ, curtailed the impact of the observations on the understanding of acoustical cavity cloud evolution, particularly over the first few hundred cycles of ultrasound exposure. Laser-induced cavitation studies allow much higher temporally and spatially resolved dynamics, but have limited relevance to acoustic cavitation, as the pulse energy required to induce optical breakdown in the host medium result in cavity dimensions typically above a few $100 \mu \mathrm{m}$, much larger than those typically encountered in $\mathrm{MHz}$ ultrasound fields at cavitation inception. In this paper, we report the development of an instrument that combines conventional approaches to study acoustic and optical cavitation, and which permits the use of high-speed cameras at $\mathrm{MHz}$ frame rates to observe the evolution of acoustically driven cavitation clouds. Laser-induced cavitation in an ultrasound field has been previously investigated using high-speed photography. ${ }^{13}$ To enhance cavitation collapse phenomena, bubble luminescence was studied as a function of seeding phase in a $44.6 \mathrm{kHz}$ field. The current work is quite distinct in terms of employing low-energy nanosecond pulses in a well-characterized, moderate- to highMI focused field. Section II describes in detail the experimental arrangement and the custom-designed chamber that allows optical access to the focal region of an ultrasound bowl transducer, while also allowing the ultrasound itself to propagate unimpeded. Section III presents sample results for each of the cavitation regimes possible with the instrument including conventional laser-induced cavitation, laser-induced cavitation in a pre-established ultrasound field, and the new phenomenon of laser-nucleated acoustical cavitation.

\section{METHODOLOGY}

In this section the experimental setup developed to observe distinct regimes of hybrid acoustic and optical cavitation is described. Figure 1 is a schematic representation of the apparatus. The ultrasound was generated by a focused bowl transducer and accompanying electronics. The optical system was a standard laser-induced cavitation arrangement. The key feature of the instrument was the sonoptic chamber as shown in Fig. 2, which permits irradiation of the ultrasound focus with a laser pulse, focused through a long working distance objective lens without disrupting the field, other than intentionally with cavitation activity. The entire device was constructed on an RS2000 active self-leveling and vibration damping optical table (Newport, Didcot, Oxfordshire, UK).

\section{A. Acoustics}

\section{Focused ultrasound}

The ultrasound source was a single-element, $100 \mathrm{~mm}$ diameter, piezoelectric transducer (PZT), spherically focused ultrasound transducer with a geometric focus of $80 \mathrm{~mm}$ (GE Healthcare, Waukesha, WI). The transducer had an efficiency of $30 \%$ at its impedance-matched resonance frequency of $1.47 \mathrm{MHz}$. The device was driven by an AFG3102 (Tektronix, Everett, WA) arbitrary function generator. The signal was passed via a $20 \mathrm{~dB}$ attenuator to a 3100LA, 55 dB RF amplifier (Electronics \& Innovation, Rochester, NY). The ultrasound field generated was characterized, cf. Fig. 3, in a custom-made three-dimensional scanning tank, using a $200 \mu \mathrm{m}$ PVDF needle hydrophone (Onda, Sunnyvale, CA). These free-field profiles were subsequently used to validate the field when the transducer was located within the sonoptic chamber. The custom-made sonoptic chamber shown in Fig. 2 was designed and constructed specifically for this transducer so as the ultrasound beam produced could propagate through the focus without scattering or reflection. The transducer housing was constructed out of $6 \mathrm{~mm}$ thick polycarbonate sheets and had internal dimensions of $188 \times 188 \times 89 \mathrm{~mm}^{3}$. Above the transducer housing, two polyvinyl chloride funnels connected via the tapered ends to a $20 \times 20 \times 14 \mathrm{~mm}^{3}$ glass cavitation chamber, constructed from standard $155 \mu \mathrm{m}$ thick microscope coverslips (Scientific Laboratory Supplies Ltd., Hessel, East Riding of Yorkshire, UK) and glued using Rapid ARALDITE $^{\mathrm{TM}}$ epoxy (Bostik S.A., Paris la Défense, France). The position of the cavitation chamber over the transducer

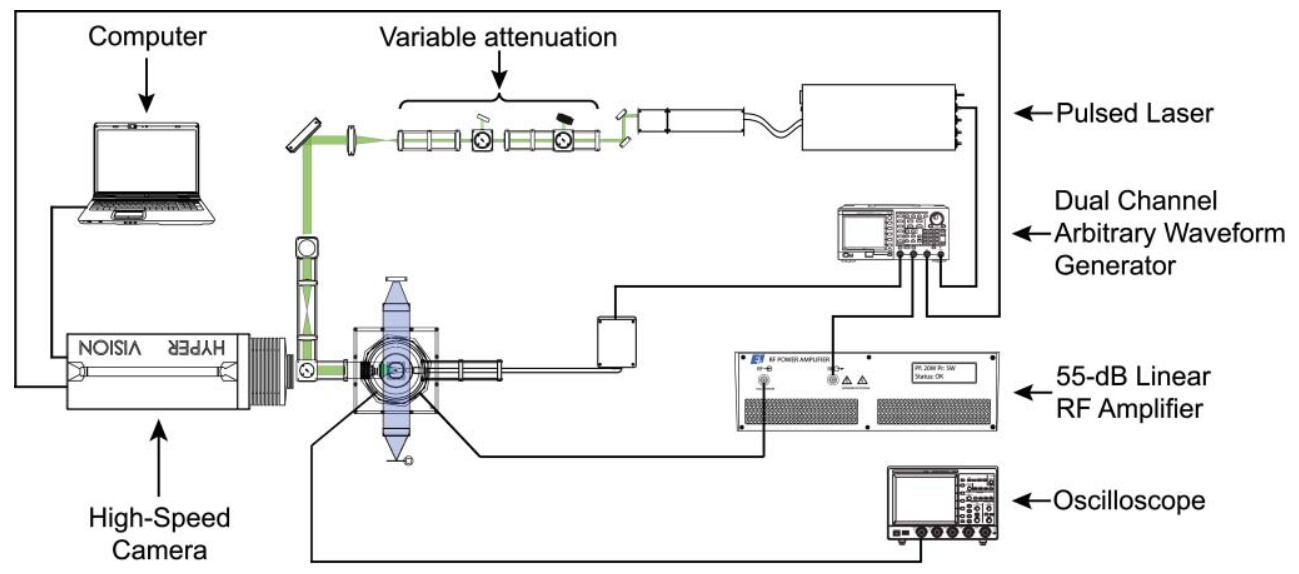

FIG. 1. (Color online) Schematic overview of apparatus, including BNC trigger lines and ultrasound electronics. 


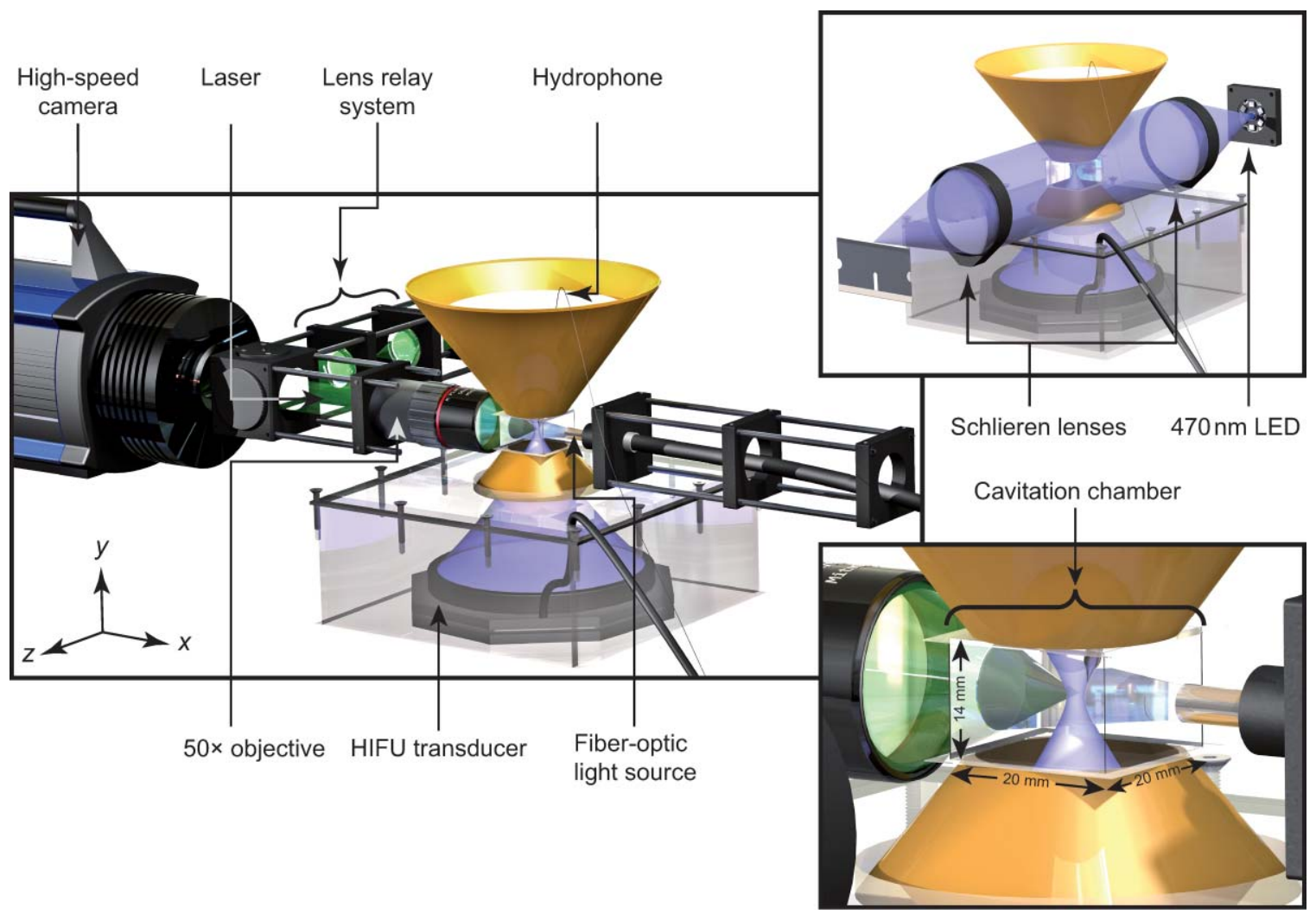

FIG. 2. (Color online) Representation of the custom-made sonoptic chamber, constructed according to the dimensions of the focused ultrasound field. The Shimadzu HPV-1 high-speed camera is depicted. Inset top right is the Schlieren imaging arrangement for alignment of the ultrasonic and optical foci. Inset bottom right is a close up of the cavitation chamber which contains the ultrasound (blue) and laser (green) foci.

was such that the center aligned with the acoustic focus. The second inverted funnel was positioned above the cavitation chamber to allow unhindered acoustic propagation into the far field. The sonoptic chamber was mounted on an M-652 $x-y-z$ microtranslation stage (Newport) for alignment of the ultrasonic focus to the optical focus.

To verify the sonoptic chamber did not impede or scatter the acoustic beam at any point, pressure maps were taken in a plane across the focal region within the cavitation chamber, Fig. 4, using a fiber optic hydrophone (Precision Acoustics, Dorset, Dorchester, UK), ${ }^{14}$ with a tapered tip of sensitivity $175 \mathrm{mV} \mathrm{MPa}^{-1}$, at $1.5 \mathrm{MHz}$.

\section{Acoustic detection}

A custom-made $200 \mu \mathrm{m}$ PZT hydrophone connected via an amplifier $(42 \times$ at $1 \mathrm{MHz})$ to an MSO7104A oscilloscope (Agilent Technologies UK Limited, Wokingham, Berkshire, UK) was situated within the cavitation chamber, outside the acoustic focus as depicted in Fig. 5(a). Figure 5(b) shows spectra generated at (i) $\mathrm{MI}=0.6$ and (ii) $\mathrm{MI}=5.5$, from the hydrophone in this position, without laser-pulse generation. At low MI, the spectral content is concentrated around the fundamental at $1.47 \mathrm{MHz}$, with the additional harmonics at high MI indicating the presence of stable cavitation, as may be anticipated in this acoustic regime. For the acoustic detection results presented below, a notch filter was used to suppress the fundamental signal from the primary field. In all experiments degassed tap water was used. This is defined as water with a oxygen content of $<4 \mathrm{mg} \mathrm{L}{ }^{-1} .{ }^{15}$ Degassing was achieved using liquid heating. ${ }^{16}$ The gas content was measured before and after the experiments using a DO 110 dissolved oxygen meter (Oakton, Vernon Hill, IL), and determined to be less than $4 \mathrm{mg} \mathrm{L}^{-1}$ for all reported experiments.

\section{B. Optics}

\section{Laser source}

The laser source was a Nano S 130-10 Q-switched Nd:YAG pulsed laser (Litron Lasers, Rugby, Warwickshire, UK) emitting up to $70 \mathrm{~mJ}$ at $532 \mathrm{~nm}$ with a pulse duration of 6-8 ns and repetition rate of up to $10 \mathrm{~Hz}$. To accurately control the pulse energy a 650-1050 nm, $\varnothing 10 \mathrm{~mm}$, GL10-B, antireflection coated, polarizing cubes (Thorlabs, LTD., Ely, Cambridgeshire, UK) and $532 \mathrm{~nm}, \varnothing 12.7 \mathrm{~mm} \lambda / 2$-wave plates (Thorlabs) were used. The first cube and wave-plate pair provided coarse attenuation and the second was used for fine tuning. The beam was expanded to slightly overfill the back aperture of the objective lens, ensuring diffraction limited focal volume. This was achieved with a two times expansion telescope constructed from $f_{150 \mathrm{~mm}}$ and $f_{300 \mathrm{~mm}}$ antireflection coated lenses. The pulse was redirected from a $532 \mathrm{~nm}$ $\varnothing 25.4 \mathrm{~mm}$ dichroic mirror (Thorlabs) into the back aperture of the objective. This permits high-speed observation through the same objective as depicted in Fig. 2. To control beam collimation prior to entering the objective lens, the pulse was 
(a)

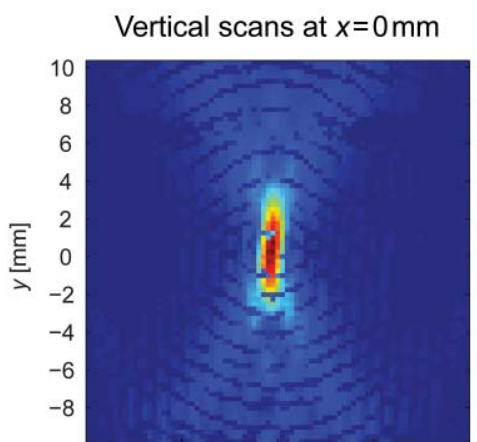

(b)

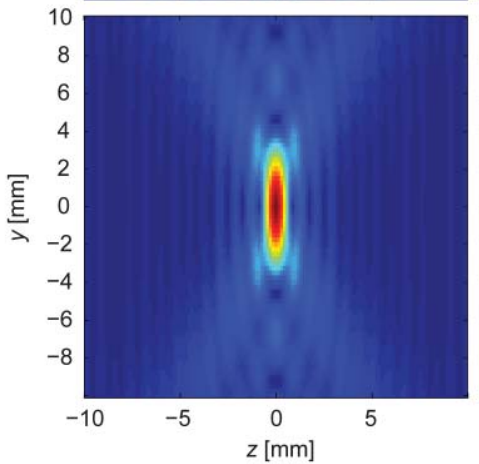

Horizontal scans at $y=0 \mathrm{~mm}$
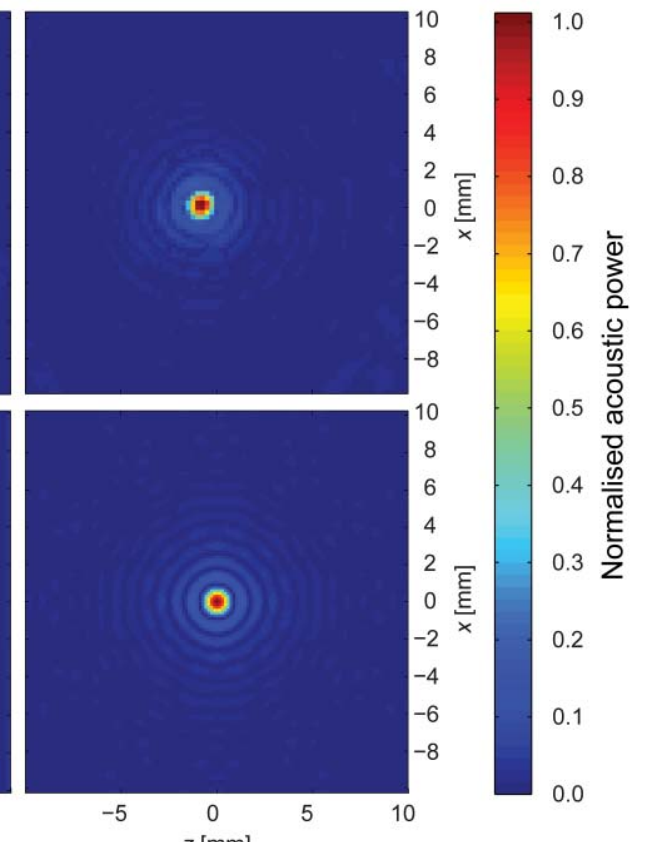

FIG. 3. (Color online) (a) Scanned axial and transverse pressure fields of the focused bowl transducer used as the ultrasound source throughout the experiments, recorded in an ultrasound scanning tank. (b) Simulation of field based on the dimensions of the transducer.

passed through a $4 f$ lens relay system comprised of two $f_{150 \mathrm{~mm}}$ conjugate lenses. Two objective lenses were used for the observations presented, including $5 \times 0.14$ NA, M Plan APO, infinity corrected lenses (Mitutoyo, Aurora, IL), and a $50 \times 0.42$ NA, M Plan, NIR, infinity corrected lens (Mitutoyo). The laser energy was measured at the back aperture of the objective using a S370C power meter (Thorlabs). Laser beam steering was achieved using $\varnothing 25.4 \mathrm{~mm}$ and $\varnothing 76.2 \mathrm{~mm}$ protected silver mirrors (Thorlabs). The high-speed camera was protected from scattered laser radiation using a dichroic band-pass filter 540 IB 50, >98\% at $532 \mathrm{~nm}$ (Comar Instruments, Cambridge, UK).

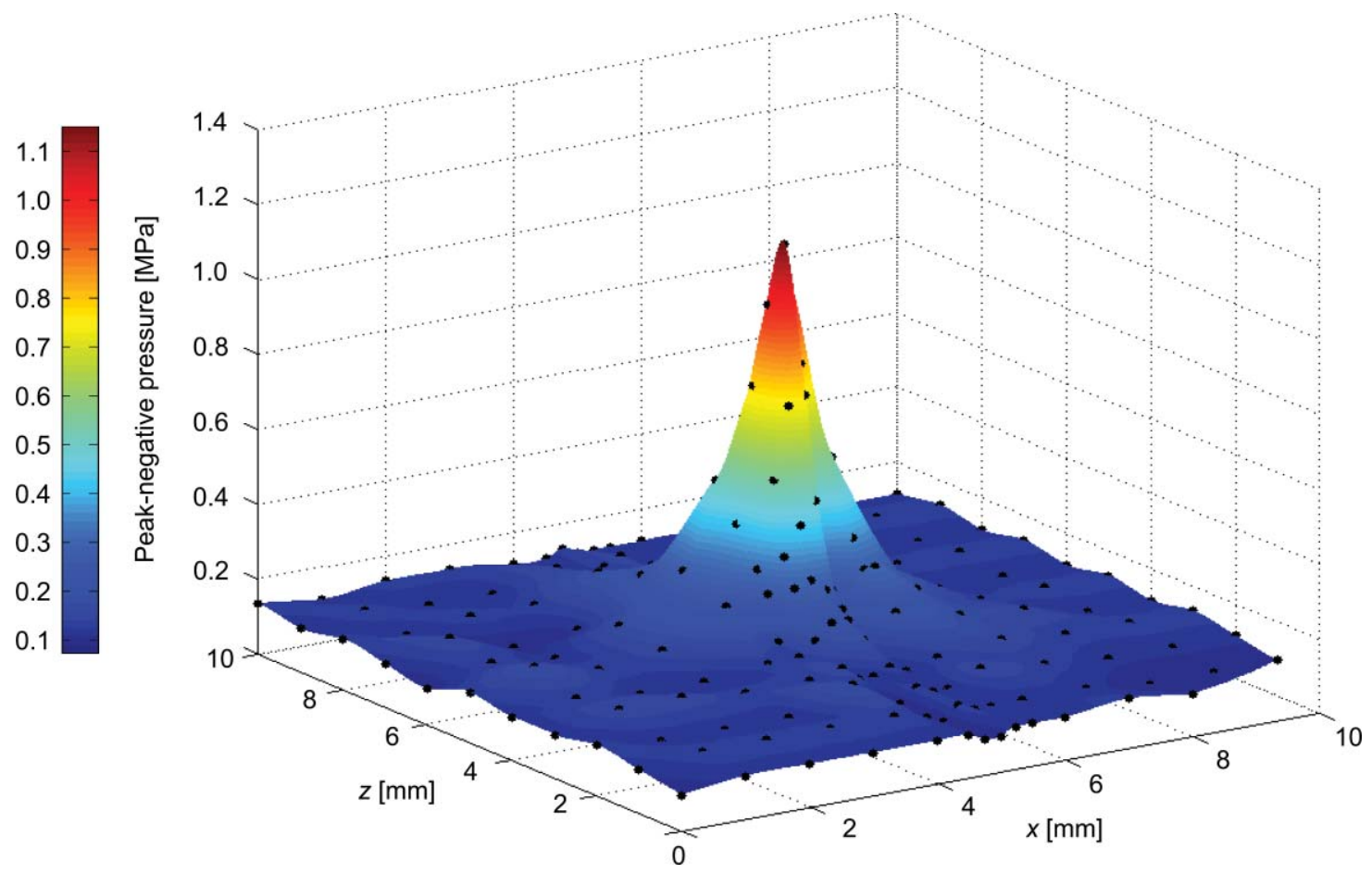

FIG. 4. (Color online) Cubic interpolation of the two-dimensional scans, with sample pressure data measured in the acoustic focus within the sonoptic chamber (black dots), demonstrating ultrasound propagation. 

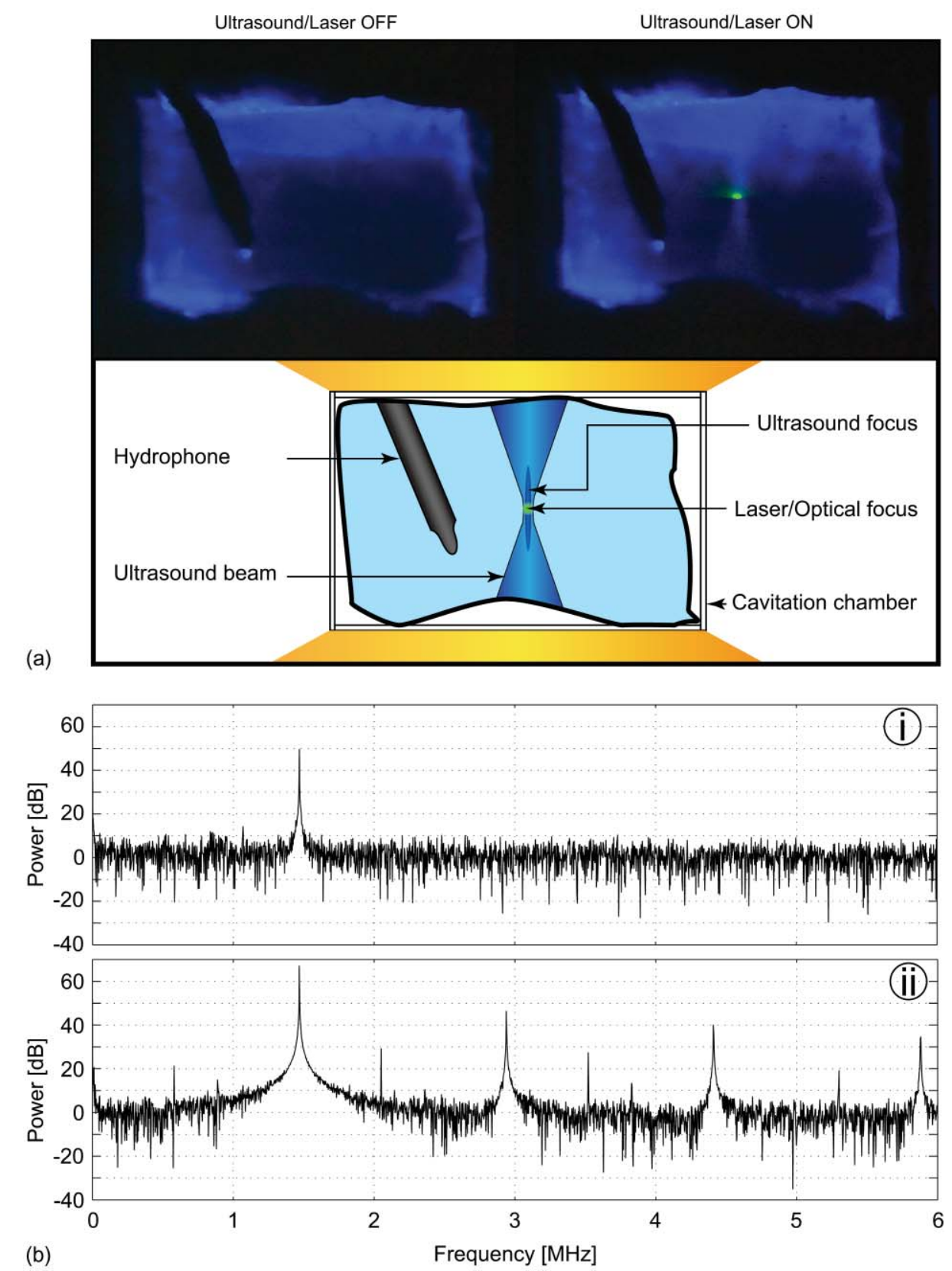

FIG. 5. (Color online) (a) Schlieren image of cavitation chamber, used to align the laser focus (green spot) to that of the ultrasound (lighter blue region). The omnidirectional needle hydrophone used to record acoustical emissions during cavitation activity is also visible. (b) Fourier spectra of sound field where (i) $\mathrm{MI}=0.6$ and (ii) $\mathrm{MI}=5.5$.

\section{Schlieren imaging}

Alignment between the acoustic and optical foci is critical to obtain reproducible results. Therefore, a monochromatic Schlieren imaging setup was built around the optical windows of the cavitation chamber, ${ }^{17}$ orthogonal to the laser propagation axis. Schlieren imaging allows for the visualization of density, or pressure, variations within transparent media. ${ }^{18}$ A 30 lm, Lambertian Luxeon V Star, $470 \mathrm{~nm}$ lightemitting diode (Lumileds Lighting, LLC, San Jose, CA) was used as a continuous light source in combination with two $\varnothing 50.8 \mathrm{~mm}, f_{150} \mathrm{~mm}$ lenses (Comar Instruments) to generate a collimated beam across the optical window. Large lenses were used to fully cover the cavitation chamber. A razor blade was mounted on a vertical translation slide to provide the zero-order stop, while the image was projected onto a white screen. A Schlieren shadowgraph showing the laser spot and the acoustic focus during the alignment procedure is shown in Fig. 5(a). The shadow of the needle hydrophone used to record acoustical data during cavitation experiments is also apparent in this image.

\section{HIGH-SPEED PHOTOGRAPHY}

High-speed cameras may be incorporated into the experiment via one of the two possible configurations. As depicted in Figs. 1 and 2, imaging can be achieved through the same objective that focuses the laser into the ultrasound field. There is also the option of imaging through a second objective lens, orthogonal to the laser propagation direction, 


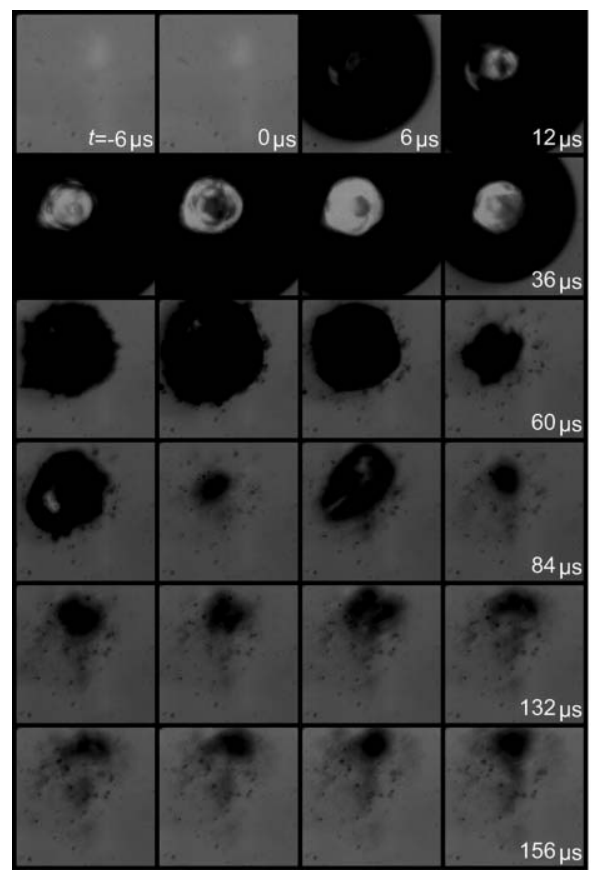

(a)

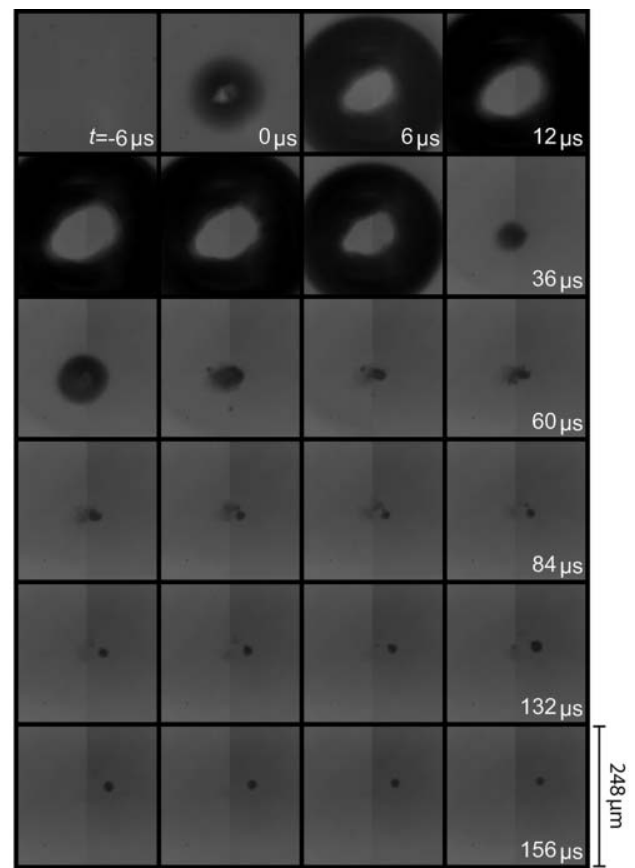

(b)

FIG. 6. Cavitation recorded with the HPV-1 high-speed camera at $0.5 \mathrm{Mfps}$, with simultaneous imaging and focusing the laser pulse through the $50 \times$ objective lens. (a) Laser-induced cavitation from a $1.2 \mathrm{~mJ}$ laser pulse. (b) Laser-induced cavitation in a pre-established focused ultrasound field of $1.47 \mathrm{MHz}$ and $\mathrm{MI}=1.1$. Each frame corresponds to a $248 \times 248 \mu \mathrm{m}^{2}$ area.

once the Schlieren optics have been removed, following the alignment procedure. The fiber optic bundle delivering the flash illumination to the cavitation chamber must also be rotated through $90^{\circ}$, from the position represented in Figs. 1 and 2. This configuration offers the additional advantage of image focusing independently from that of the laser pulse. The choice of high-speed camera is largely determined by the frame rate required to observe a particular cavitation-related phenomenon. In this paper we present results obtained with two high-speed cameras: a Shimadzu HPV-1 (Shimadzu, Kyoto, Japan), and a Cordin Model 550-62 device (Cordin, Salt Lake City, UT). The former consists of a single CCD sensor capable of recording 100 frames of $312 \times 260$ pixels at frame rates up to $1 \times 10^{6}$ frames per second (Mfps) at minimum exposure times of $125 \mathrm{~ns}$. The latter is a gas-driven, rotating mirror camera capable of recording 62 frames of $1000 \times 1000$ pixels at frame rates up to $4 \mathrm{Mfps}$ when using compressed helium gas to rotate the turbine. The Model 550-62 is capable of minimum exposure times of 250 ns. For high-speed camera data acquisition rates that did not necessitate flash illumination, an LB60 continuous fiber optic light source (Welch Allyn, Skaneateles Falls, NY) coupled to a $\varnothing 4 \mathrm{~mm}$ fibreoptic cable; capable of providing $7.96 \times 10^{6}$ lux at the entry surface of the fibre-optic cable was used. For higher acquisition rates, a Model 659 (Cordin) Xenon flash system was used, coupled to the fiber optic bundle via a condenser lens (Comar Instruments). The flash-head provided $3.23 \times 10^{6}$ lux at $3 \mathrm{~m}$, with adjustable duration of up to $1 \mathrm{~ms}$. The exit end of the fiber optic cable was positioned $2 \mathrm{~mm}$ from the surface of the cavitation chamber. To ensure synchronization of the laser pulse, flash illumination, and high-speed camera operation, each component was electronically triggered with ap- propriately delayed TTL pulses. In the results presented below, $t=0 \mu \mathrm{s}$ is defined as the frame at which the laser pulse is incident to the cavitation chamber. The pretrigger option on the high-speed cameras was used to trigger the waveform generator providing the sinusoid for the ultrasound, which in turn sent two trigger pulses to the laser at $t=-120 \mu \mathrm{s}$, to account for the Q-switch delay and to the flash capacitor bank at $t=-70 \mu \mathrm{s}$, to allow the intensity to rise for maximum illumination. High-speed camera operation was set internally to $t=-10 \mu$ s to capture a number of frames before cavitation activity was initiated.

\section{RESULTS AND DISCUSSION}

At the mechanical indices used here, no cavitation was observed within the field-of-view of the high-speed cameras, prior to laser pulse irradiation.

Figure 6 provides representative images extracted from high-speed sequences recorded at $0.5 \mathrm{Mfps}$ of laser-induced cavitation. Figure 6(a) demonstrates conventional, plasmamediated cavitation, whereby optical breakdown resulted from absorption of a $1.2 \mathrm{~mJ}$ laser pulse, above the threshold pulse energy required to generate a cavity. This threshold energy was determined to be $1.1 \mathrm{~mJ}$ for our system. Rapid expansion resulted in the $248 \times 248 \mu \mathrm{m}^{2}$ field-of-view becoming overfilled, within the first few microseconds after absorption, through to $36 \mu \mathrm{s}$, when the cavity had entered a collapse phase. This was followed by a number of rebound inflations, at 48, 66, and $78 \mu \mathrm{s}$, driven by the inertia of the liquid and compressibility of the gas. During these oscillations microbubble debris formed, and moved with the liquid surrounding the oscillating primary cavity. The cavity translated 
upward from 66-84 $\mu$ s. This may be associated with the asymmetry of the inflation at $78 \mu \mathrm{s}$. Otherwise, the geometric center of the activity remained static throughout the event, since buoyancy effects are negligible over these timescales.

Figure 6(b) shows an event where the same laser-pulse energy was incident to the focal region of a pre-established ultrasound field of $M I=1.1$. Similar to Fig. 6(a), the initial $36 \mu$ s of the sequence were dominated by the rapid expansion and subsequent collapse of the laser-induced cavity. A single secondary inflation was observed at $42 \mu \mathrm{s}$, of a maximum diameter notably smaller than at the equivalent time in Fig. 6(a). Further reinflations were suppressed by the pres-

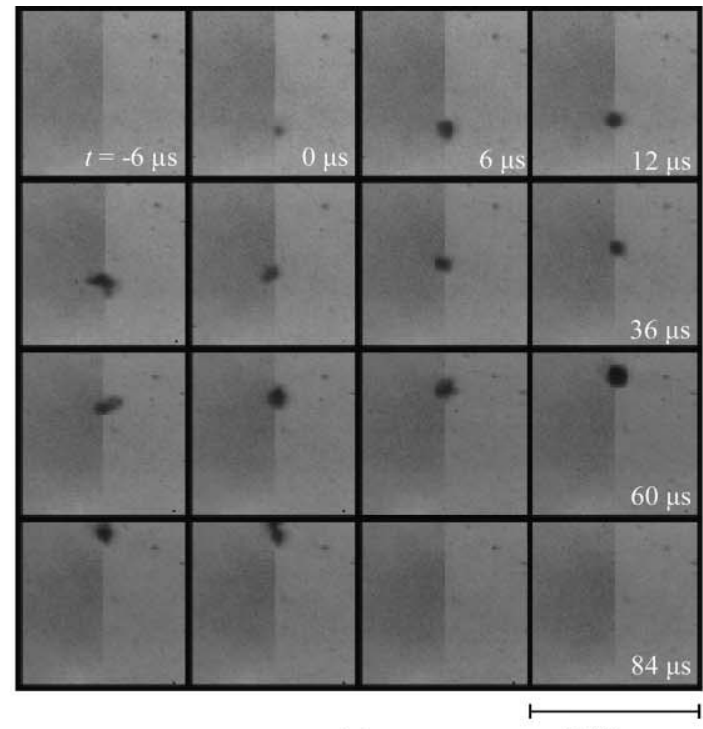

(a)

$$
248 \mu \mathrm{m}
$$

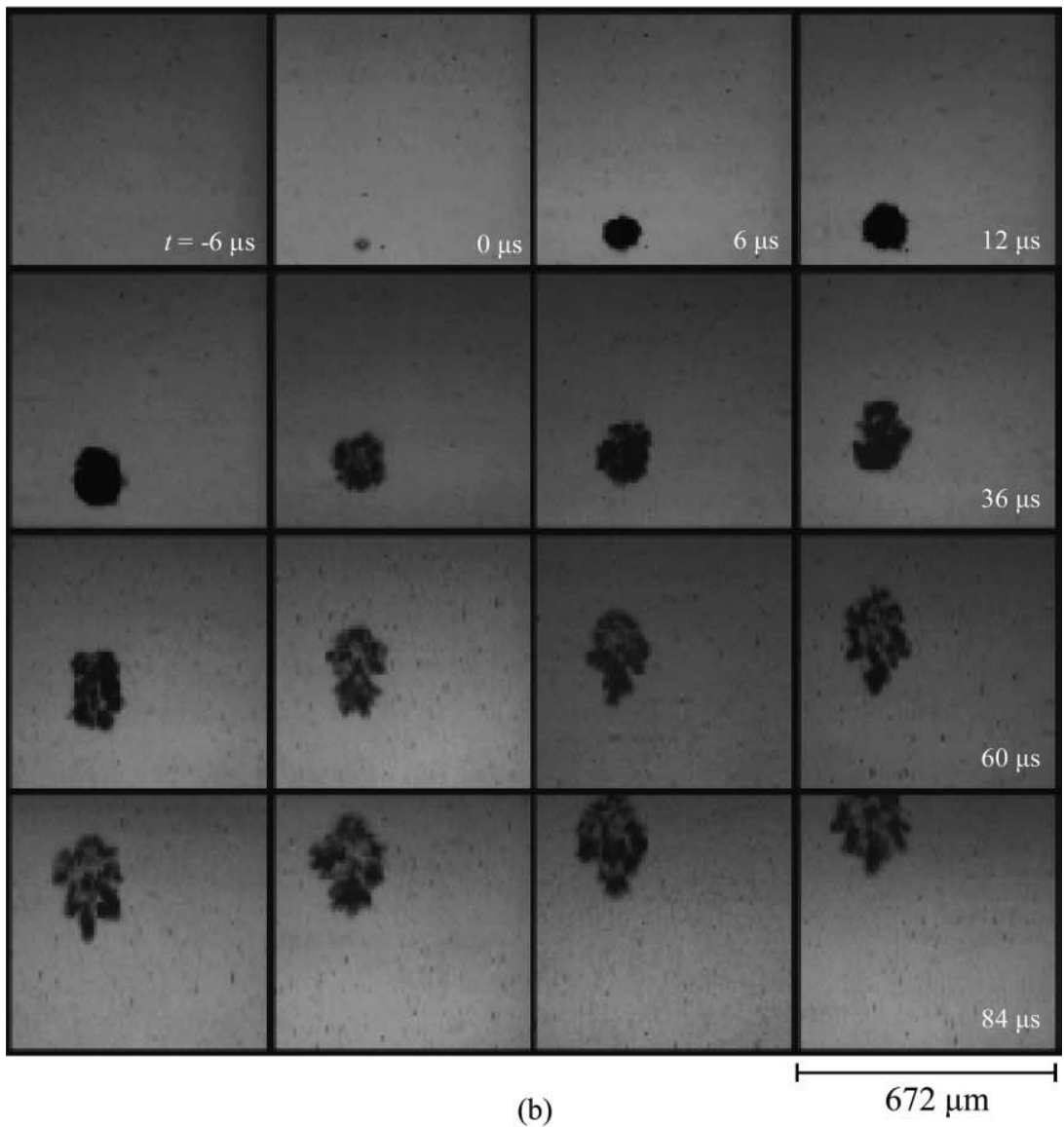

FIG. 7. (a) Laser-nucleated acoustic cavitation recorded with the Shimadzu HPV-1 camera at $0.5 \mathrm{Mfps}$ with simultaneous imaging and focusing the laser pulse through the $50 \times$ objective lens. A $0.95 \mathrm{~mJ}$ laser pulse was focused into the focal volume of the ultrasound field of identical parameters to that of Fig. 6(b). Each frame corresponds to a $248 \times 248 \mu \mathrm{m}^{2}$ area. (b) Laser-nucleated cavitation in a field of higher MI $=3.4$, recorded with the Cordin $550-62$ high-speed camera at $0.5 \mathrm{Mfps}$. For this sequence, imaging was performed through the $5 \times$ objective, in the orthogonal configuration described in Sec. III, to achieve a larger field-of-view of $672 \times 672 \mu \mathrm{m}^{2}$. The laser pulse was focused through the $50 \times$ objective. 


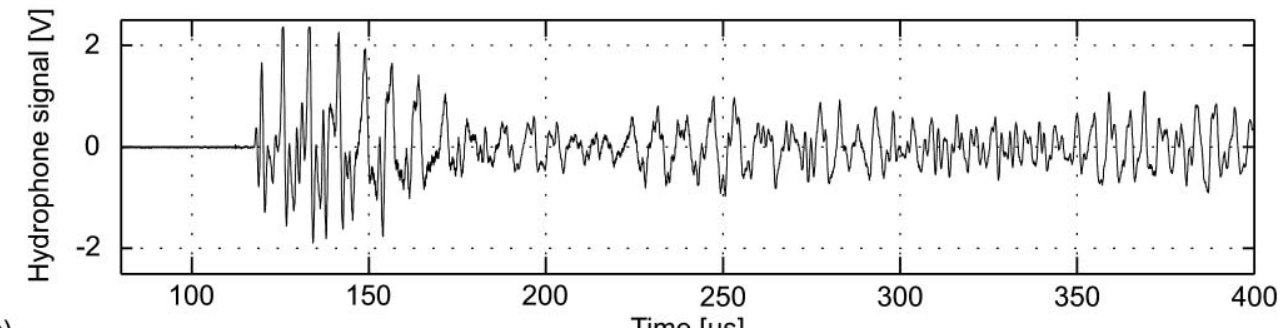

(a)

Time $[\mu \mathrm{s}]$
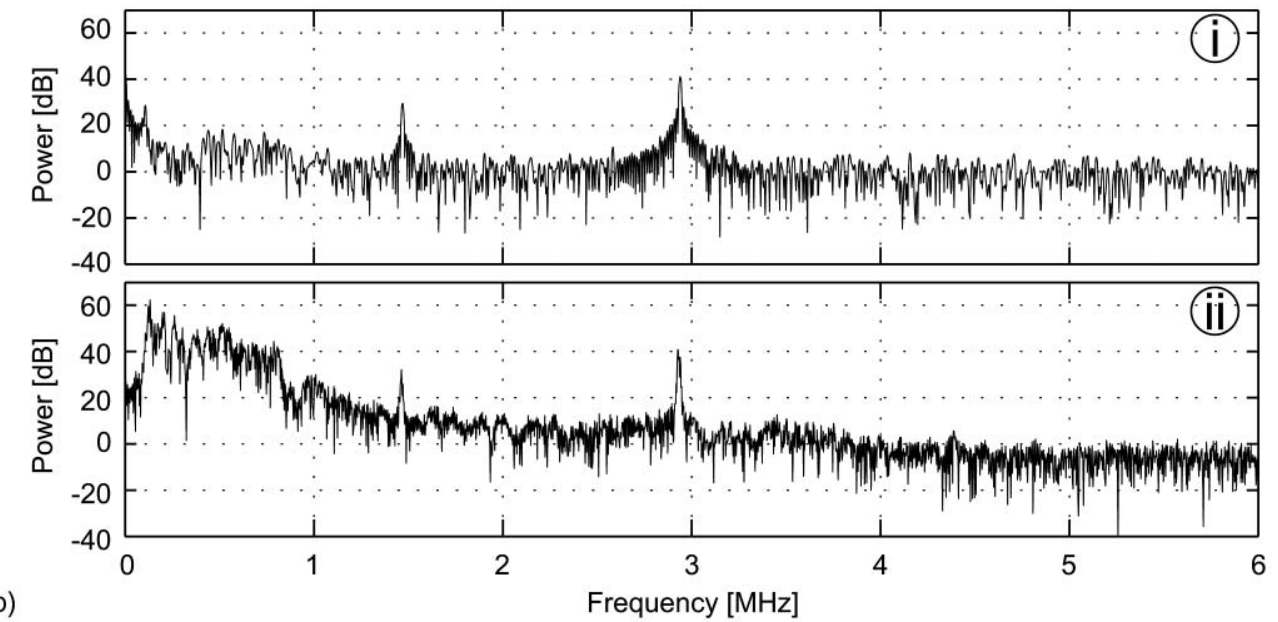

FIG. 8. (a) The acoustic signal recorded from the hydrophone positioned within the cavitation chamber, as depicted in Fig. 5, notch-filtered at 1.47 MHz, recorded during the high-speed images of laser-nucleated cavitation depicted in Fig. 7(b). (i) The spectra of the first $120 \mu$ s of the signal and (ii) the remaining $280 \mu \mathrm{s}$, during laser-nucleated acoustic cavitation.

ence of the ultrasound field, as was the level of microbubble debris. At $60 \mu \mathrm{s}$, a small cloud of debris started to translate upward, in the direction of ultrasound propagation, owing to primary radiation forces, ${ }^{19,20}$ at an average velocity of $1.0 \mathrm{~m} \mathrm{~s}^{-1}$. The formation of the cavitation clouds themselves is also determined by secondary radiation (Bjerknes) forces. ${ }^{19-21}$ For a complete review on cloud dynamics, we refer to Section 12 of Lauterborn and Kurz. ${ }^{22}$

Figure 7(a) represents a process we have termed laser-nucleated acoustic cavitation, to distinguish it from laser-induced cavitation in an ultrasound field such as that of Fig. 6(b). Here, a $0.95 \mathrm{~mJ}$ laser pulse, which is below the optical cavitation threshold for our system under ambient pressure conditions, nucleated cavitation activity in the preestablished ultrasound field. Rather than the rapid expansion of the laser-induced cavity, to a maximum diameter of a few hundred micrometers, a microcavity of diameter of $<14 \mu \mathrm{m}$ was formed at $t=0 \mu \mathrm{s}$. It translated owing to primary radiation forces, at an average velocity of $1.5 \mathrm{~m} \mathrm{~s}^{-1}$, while undergoing volumetric oscillations.

Figure 7(b) represents selected images from a sequence of laser-nucleated acoustic cavitation in an ultrasound field of significantly increased acoustic pressure $(\mathrm{MI}=3.4)$ with a $0.95 \mathrm{~mJ}$ laser pulse. Here, a single cavity of diameter $<25 \mu \mathrm{m}$ formed at $t=0 \mu \mathrm{s}$ and initiated what later revealed itself to be a cavitation cloud: a larger number of distinct cavities, interacting, coalescing, and reforming, but also exhibiting collective behavior as a single entity. ${ }^{19,20}$ The cloud retained sphericity for $18 \mu \mathrm{s}$, but elongated in the direction of the ultrasound propagation axis from $36 \mu \mathrm{s}$, before adopting a mushroom-shaped morphology at $54 \mu \mathrm{s}$, reminiscent of the caplike structure reported previously. ${ }^{12}$ These morphology changes were accompanied by rapid translation of the cloud, owing to primary radiation forces with an average velocity of $7.0 \mathrm{~m} \mathrm{~s}^{-1}$.

Figure 8(a) shows the hydrophone signal recorded during the laser-nucleated acoustic cavitation of Fig. 7(b), with the laser pulse incident to the ultrasound focus at $t=120 \mu \mathrm{s}$. The sudden increase in signal corresponds to high-speed observations of the onset of cavitation activity, which decays as the cluster translates away from the hydrophone tip position, under the primary radiation force. Figure 8(b) (i) shows the spectrum of the notch-filtered hydrophone signal of the first $120 \mu$ s, i.e., the signal of the pre-established ultrasound field. The presence of a second harmonic peak and the absence of broadband emissions suggest stable cavitation outside the field-of-view before the laser pulse had been generated. Figure 8(b) (ii) represents the spectrum of the hydrophone signal from 120-400 $\mu$ s, which exhibits harmonic. In addition, a strong increase in acoustic emissions from $100 \mathrm{kHz}$ to $1 \mathrm{MHz}$ is evident. This suggests that the laser-nucleated acoustic cavitation of Fig. 7(b) is inertial.

\section{CONCLUSION}

We have demonstrated that acoustic cavitation can be forced to occur at a specific location in a liquid by using a low energy, nanosecond laser pulse to nucleate activity in a preestablished ultrasound field. The low pulse energy avoids the large, plasma-mediated cavities, generally associated with optical cavitation. It also permits the incorporation of high-speed cameras to image the dynamics at microsecond temporal and 
micrometer spatial resolutions. This technique will contribute to the understandings of the cavitation evolution in focused ultrasound including for potential therapeutic applications for focused ultrasound surgery.

\section{ACKNOWLEDGMENTS}

This work has been supported by European Union (EU) FP7 [Grant No. 230674 (Nanoporation)], Deutsche Forschungsgemeinschaft (DFG) Emmy Noether Programme (Grant No. 38355133), and Engineering and Physical Sciences Research Council (United Kingdom) [EPSRC(GB)] (Grant Nos. EP/G01213X/1 and EP/F037025/1). Bjoern Gerold has been supported by an EPSRC DTA award. David McGloin is supported by the Royal Society. We are very grateful to EPSRC loan pool, notably to Adrian Walker, for ongoing access to high-speed imaging devices; to Javier Grinfeld, Yoav Medan, Oleg Prus, and Alex Volovick, all from InSightec Ltd, Tirat Carmel, Israel, for ongoing technical support; and Joyce Joy for ultrasound calibration measurements.

${ }^{1}$ F. R. Young, Cavitation (McGraw-Hill, Maidenhead, UK 1989).

${ }^{2}$ C. K. Holland and R. E. Apfel, IEEE Trans. Ultrason. Ferroelectr. Freq. Control 36, 204 (1989).

${ }^{3}$ C. Coussios, C. H. Farny, G. R. ter Haar, and R. Roy, Int. J. Hyperthermia 23, 105 (2007)
${ }^{4}$ R. Holt and R. Roy, Ultrasound Med. Biol. 27, 1399 (2001).

${ }^{5}$ T. G. Leighton, The Acoustic Bubble (Academic, London, UK, 1994).

${ }^{6}$ M. Postema and G. Schmitz, Expert Rev. Mol. Diagn. 6, 493 (2006).

${ }^{7}$ W. Hentschel and W. Lauterborn, Appl. Sci. Res. 38, 225 (1982).

${ }^{8}$ J. Fujimoto, W. Lin, E. Ippen, C. Puliafito, and R. Steinert, Invest. Ophthalmol. Visual Sci. 26, 1771 (1985). http://www.iovs.org/ content/26/12/1771.full.pdf

${ }^{9}$ C. D. Ohl and E. Ory, in Nonlinear Acoustics at the Turn of the Millennium, edited by W. Lauterborn and T. Kurz (American Institute of Physics, New York, 2000), pp. 393-396.

${ }^{10}$ A. Philipp and W. Lauterborn, J. Fluid Mech. 361, 75 (1998).

${ }^{11}$ H. Chen, L. Xiaojing, M. Wan, and S. Wang, Ultrason. Sonochem. 14, 291 (2007).

${ }^{12} \mathrm{H}$. Chen, X. Li, and M. Wan, Ultrason. Sonochem. 13, 480 (2006).

${ }^{13}$ T. Kurz, D. Kröninger, R. Geisler, and W. Lauterborn, Phys. Rev. E 74, 066307 (2006).

${ }^{14}$ P. Morris, A. Hurell, A. Shaw, E. Zhang, and P. Beard, J. Acoust. Soc. Am. 125, 3611 (2009).

${ }^{15}$ A. Shaw and G. R. ter Haar, "Requirements for measurements standards in high intensity focused ultrasound (HIFU) fields," Tech. Rep. DQL AC 015 (National Physical Laboratory, February, 2006).

${ }^{16}$ J. Fowlkes and P. Carson, J. Acoust. Soc. Am. 90, 1197 (1991).

${ }^{17}$ T. Neumann and H. Ermert, Ultrasonics 44, e1561 (2006).

${ }^{18} \mathrm{C}$. Alvarez-Herrera, D. Moreno-Hernández, B. Barrientos-García, and J. Guerrero-Viramontes, Opt. Laser Technol. 41, 233 (2008).

${ }^{19}$ T. Leighton, Ultrason. Sonochem. 2, S123 (1995).

${ }^{20}$ S. Kotopoulis and M. Postema, Ultrasonics 50, 260 (2010).

${ }^{21}$ R. Mettin, I. Akhatov, U. Parlitz, C. D. Ohl, and W. Lauterborn, Phys. Rev. E 56, 2924 (1997).

${ }^{22}$ W. Lauterborn and T. Kurz, Rep. Prog. Phys. 73, 106501 (2010). 